\title{
Sistemas Frontais sobre a América do Sul Parte II: Monitoramento Mensal em dados da Reanálise I do NCEP/NCAR
}

Frontal Systems over South America Part II: Mensal Tracking for NCEP/NCAR Reanalysis I data

Luana Albertani Pampuch ${ }^{1}$ e Tércio Ambrizzi²

1,22Departamento de Ciências Atmosféricas, Universidade de São Paulo, São Paulo, Brasil

lupampuch@gmail.com; tercio.ambrizzi@iag.usp.br

\begin{abstract}
Resumo
Um monitoramento dos sistemas frontais para a América do Sul é realizada pelo Grupo de Estudos Climáticos da Universidade de São Paulo (GrEC/USP) desde janeiro de 2014. Para esta análise é utilizada uma metodologia objetiva de rastreamento dos sistemas frontais que consiste em analisar dados diários da Reanálise I do NCEP/NCAR e verificar a ocorrência de queda na temperatura em 925hPa, simultânea a um giro no vento meridional em $925 \mathrm{hPa}$ e aumento na pressão ao nível médio do mar de um dia para o outro. Este monitoramento é feito através de mapas com o número e a anomalias de sistemas frontais e também para a análise da ocorrência destes sistemas em pontos de grade sobre o litoral, interior e centro do continente.
\end{abstract}

Palavras-chave: Sistemas frontais. Monitoramento. América do Sul.

\begin{abstract}
A tracking of the frontal systems over South America is performed by the Climate Studies Group at the University of São Paulo (GrEC/USP) since January 2014. This analysis used an objective methodology for tracking the frontal systems that consists in analyze daily data from I NCEP / NCAR Reanalysis I and verify the occurrence of decrease in temperature at 925hPa, simultaneous to a shift in the meridional wind in $925 \mathrm{hPa}$ and sea level pressure increase one day to another. This monitoring is done through maps with the number and anomalies of frontal systems and also analyzing the occurrence of these systems in grid points on the coast, inland and center of the continent.
\end{abstract}

Keywords: Frontal systems. Tracking. South America. 


\section{Introdução}

A América do Sul se apresenta como uma região favorável à ocorrência de sistemas frontais, em parte, devido às características atmosféricas associadas à sua posição geográfica. Sua localização entre a ASPS (Alta Subtropical do Pacífico Sul) e a ASAS (Alta Subtropical do Atlântico Sul) favorece a intensificação de gradientes de temperatura e de deformação horizontal e criam um ambiente favorável para estes sistemas (Satyamurty e Mattos, 1989).

Os sistemas frontais ocorrem sobre a América do Sul durante todo o ano, sendo registrados em maior número sobre latitudes mais altas, e apresentam um deslocamento típico de sudoeste para nordeste (Cavalcanti e Kousky, 2009). O inverno é a estação que apresenta a maior ocorrência destes sistemas e no verão um menor registro é verificado (Andrade e Cavalcanti, 2004; Andrade, 2005; Cavalcanti e Kousky, 2009; Pampuch e Ambrizzi, 2015).

A ocorrência destes sistemas geralmente está associada à instabilidade, nuvens com grande desenvolvimento vertical, diminuição na temperatura, ventos mais fortes e precipitação (Fedorova, 1999).

Desta forma, é de extrema importância o monitoramento dos sistemas frontais, já que um melhor entendimento de como e onde eles ocorrem pode ajudar a melhorar previsões de tempo e clima sobre a América do Sul e podem ajudar a prevenir os danos materiais causados por eles.

Os centros de monitoramento de tempo geralmente utilizam métodos subjetivos para a identificação destes sistemas, ou seja, a identificação é feita manualmente (Berry et al., 2011). Este procedimento gera diferenças quando analisados os resultados de diferentes institutos, já que não é utilizada uma metodologia única.

Por exemplo, O CPTEC/INPE (Centro de Previsão de Tempo e Estudos Climáticos/Instituto Nacional de Pesquisas Espaciais) disponibilizava para o período de dezembro de 1995 a fevereiro de 2014, em seu boletim Climanálise, o número de sistemas frontais para latitudes entre $25^{\circ} \mathrm{S}$ e $35^{\circ} \mathrm{S}$. O posicionamento e deslocamento dos sistemas frontais era feito pelo Grupo de Previsão do Tempo do CPTEC/INPE utilizando os campos de convergência de umidade na camada limite, temperatura potencial equivalente e advecção de temperatura potencial equivalente em $850 \mathrm{hPa}$, além de cartas sinóticas de superfície e nos níveis de $500 \mathrm{hPa}$ e $250 \mathrm{hPa}$ para as 00 e $12 \mathrm{UTC}$ (Notas do Boletim Climanálise).

O Grupo de Estudos Climáticos da Universidade de São Paulo (GrEC/USP) realiza mensalmente o monitoramento Climático para o Brasil, sendo a identificação dos sistemas frontais ocorridos no mês de análise de extrema importância para estas investigações.

Alguns trabalhos (p. ex. Cavalcanti e Kousky, 2003, Andrade e Cavalcanti, 2004, Andrade, 2005, Cavalcanti e Kousky, 2009, Pampuch e Ambrizzi, 2015) utilizaram um método objetivo de rastreamento de sistemas frontais baseados na ocorrência de condições atmosféricas durante estes fenômenos (decréscimo na temperatura, aumento na PNMM e mudança de norte para sul na direção do vento meridional), gerando climatologias destes sistemas sobre a América do Sul.

Desta forma, o objetivo deste trabalho é utilizar o método objetivo de identificação de sistemas frontais, já citado nos trabalhos acima, para identificar os sistemas frontais mensalmente em dados da Reanálise I do National Centers for Environment Prediction/National Center for Atmospheric Research (NCEP/NCAR), gerando mapas: do número de sistemas frontais sobre a América do Sul; de anomalias da ocorrência destes sistemas baseado na climatologia mensal (1981-2010) calculada por Pampuch e Ambrizzi (2015); e da posição geográfica/data da ocorrência destes eventos em pontos de grade sobre o litoral, interior e centro do continente e disponibilizá-los no site do GrEC (http://www.grec.iag.usp.br).

\section{Dados e Metodologia}

O monitoramento mensal dos sistemas frontais utiliza dados com médias diárias da temperatura e vento meridional em $925 \mathrm{hPa}$ e da pressão ao nível médio do mar (PNMM) do conjunto de dados da Reanálise I do NCEP/NCAR, que possui resolução espacial de $2.5^{\circ} \times 2.5^{\circ}$ (Kalnay et al., 1996).

A identificação dos sistemas frontais é feita para a mesma área de estudo e com a mesma metodologia empregada em Pampuch e Ambrizzi (2015), bem como a anomalia mensal 
dos sistemas frontais é calculada com a climatologia mensal (1981-2010) proveniente da figura 2 (a-1) deste mesmo trabalho.

A data de ocorrência dos sistemas frontais é monitorada em 21 pontos de grade mostrados espacialmente no mapa da figura 1, e suas coordenadas geográficas mostradas na tabela ao lado. Entre eles, 7 pontos de grade estão sobre o litoral (pontos em vermelho), 7 sobre o interior (pontos em verde) e 7 sobre o centro do continente (pontos em roxo).
A escolha destes pontos foi feita de forma a tentar cobrir as regiões litorâneas, do interior e central do continente com o maior número de regiões para rastreamento.

Para cada mês analisado, é identificado o dia da passagem dos sistemas frontais sobre cada um destes pontos, a fim de construir um mapa da ocorrência destes sistemas, mostrando suas trajetórias.

\section{Regiões para Monitoramento de Sistemas Frontais na América do Sul}

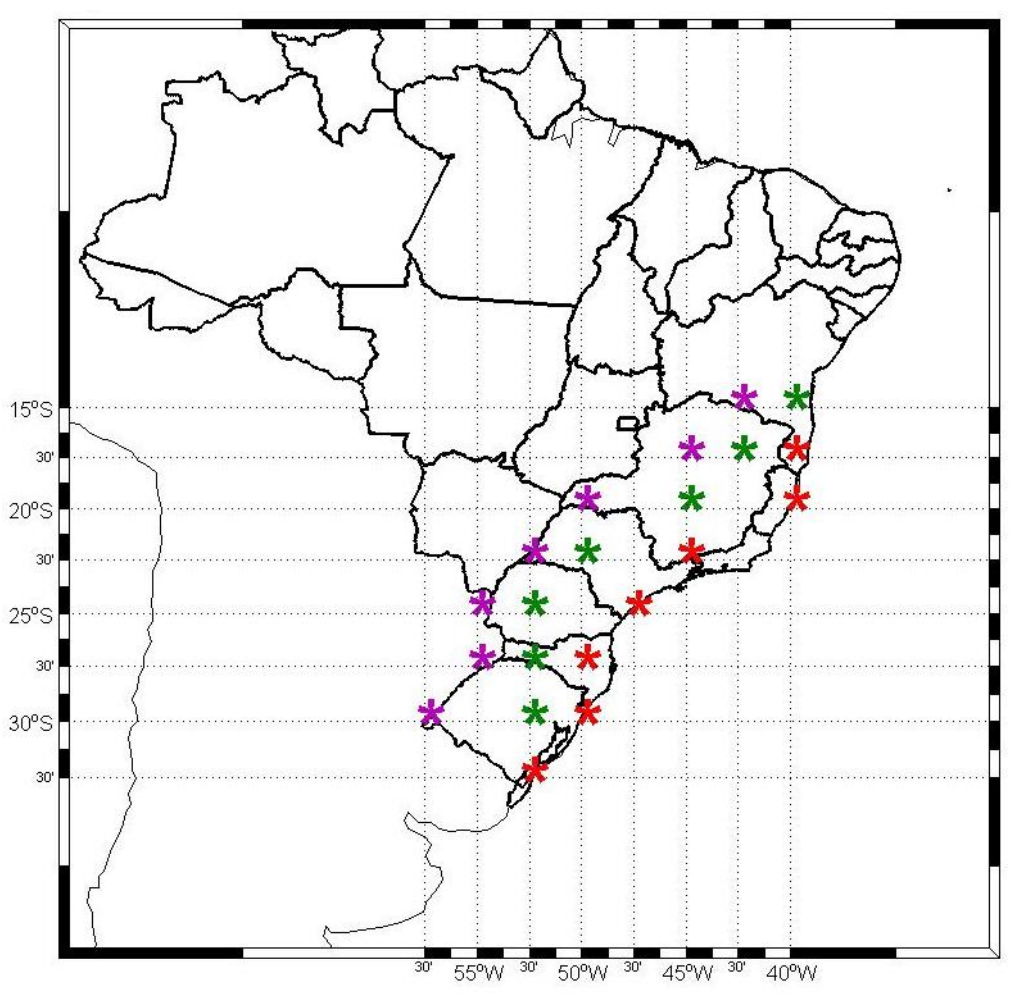

\begin{tabular}{|c|}
\hline$*$ LITORAL \\
\hline $32.5^{\circ} \mathrm{S}-52.5^{\circ} \mathrm{W}$ \\
\hline $30^{\circ} \mathrm{S}-50^{\circ} \mathrm{W}$ \\
\hline $27.5^{\circ} \mathrm{S}-50^{\circ} \mathrm{W}$ \\
\hline $25^{\circ} \mathrm{S}-47.5^{\circ} \mathrm{W}$ \\
\hline $22.5^{\circ} \mathrm{S}-45^{\circ} \mathrm{W}$ \\
\hline $20^{\circ} \mathrm{S}-40^{\circ} \mathrm{W}$ \\
\hline $17.5^{\circ} \mathrm{S}-40^{\circ} \mathrm{W}$ \\
\hline
\end{tabular}

\begin{tabular}{|c|}
\hline$*$ INTERIOR \\
\hline $30^{\circ} \mathrm{S}-52.5^{\circ} \mathrm{W}$ \\
\hline $27.5^{\circ} \mathrm{S}-52.5^{\circ} \mathrm{W}$ \\
\hline $25^{\circ} \mathrm{S}-52.5^{\circ} \mathrm{W}$ \\
\hline $22.5^{\circ} \mathrm{S}-50^{\circ} \mathrm{W}$ \\
\hline $20^{\circ} \mathrm{S}-45^{\circ} \mathrm{W}$ \\
\hline $17.5^{\circ} \mathrm{S}-42.5^{\circ} \mathrm{W}$ \\
\hline $15^{\circ} \mathrm{S}-40^{\circ} \mathrm{W}$ \\
\hline
\end{tabular}

\begin{tabular}{|c|}
\hline$*$ CENTRAL \\
\hline $30^{\circ} \mathrm{S}-57.5^{\circ} \mathrm{W}$ \\
\hline $27.5^{\circ} \mathrm{S}-55^{\circ} \mathrm{W}$ \\
\hline $25^{\circ} \mathrm{S}-55^{\circ} \mathrm{W}$ \\
\hline $22.5^{\circ} \mathrm{S}-52.5^{\circ} \mathrm{W}$ \\
\hline $20^{\circ} \mathrm{S}-50^{\circ} \mathrm{W}$ \\
\hline $17.5^{\circ} \mathrm{S}-45^{\circ} \mathrm{W}$ \\
\hline $15^{\circ} \mathrm{S}-42.5^{\circ} \mathrm{W}$ \\
\hline
\end{tabular}

Figura 1- Regiões definidas para o monitoramento mensal dos Sistemas frontais na América do Sul 


\section{Resultados e Discussões}

\subsection{Exemplo de Monitoramento}

Um exemplo de como os mapas são disponibilizados será mostrado para o mês de dezembro de 2014.

A figura 2 mostra o número de sistemas frontais identificados em dezembro de 2014 (a) e a anomalia em relação à climatologia de 19812010 (b).

É possível verificar que na região localizada entre $30^{\circ} \mathrm{S}$ e $45^{\circ} \mathrm{S}$, próximo ao centro-leste da Argentina, foi a que apresentou maior número de sistemas frontais no continente. Sobre os oceanos, o Pacífico apresentou uma região abaixo de $45^{\circ} \mathrm{S}$ em que o número de sistemas chegou a 7. Este mesmo valor foi observado no Atlântico para uma região em torno de $40^{\circ} \mathrm{S}$ na costa da Argentina e chegou a 9 sistemas na região entre $47.5^{\circ} \mathrm{S}-50^{\circ} \mathrm{S} / 20.5^{\circ} \mathrm{W}-18^{\circ} \mathrm{W}$.

Este mês mostrou uma anomalia negativa no número de sistemas frontais entre o estado do Rio Grande do Sul e o sul da Argentina, chegando a uma diminuição de até 3 sistemas frontais para estas regiões. Sobre o Oceano Atlântico Sul, próximo ao continente, esta redução também foi observada. Anomalias positivas ocorreram sobre o estado de Minas Gerais e também sobre a região do Atlântico em torno de $37.5^{\circ} \mathrm{S}-60^{\circ} \mathrm{S} / 36^{\circ} \mathrm{W}-2.5^{\circ}$. (a)

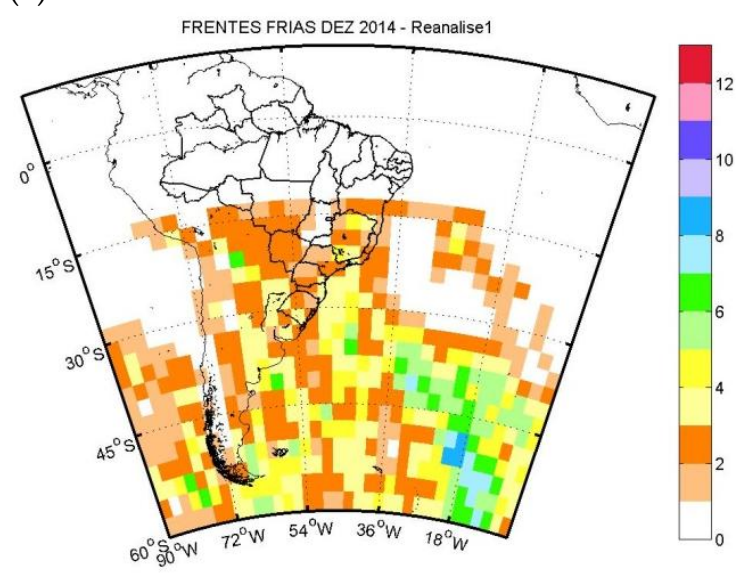

(b)

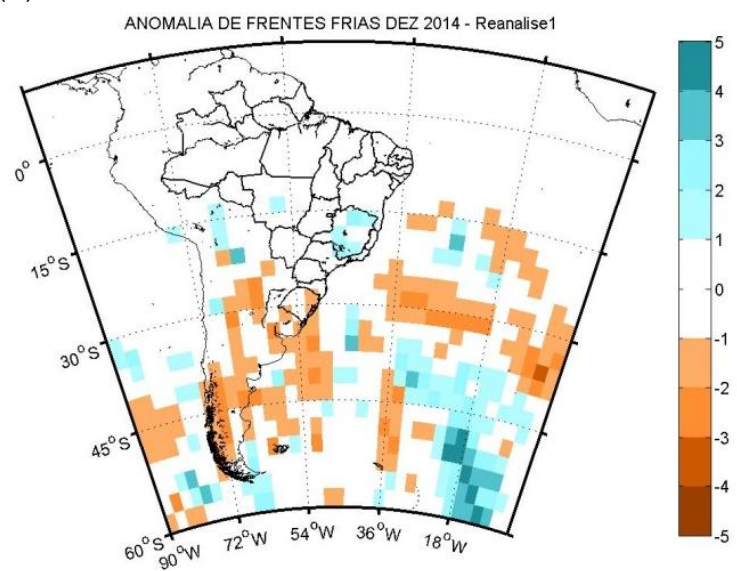

Figura 2 - Quantidade (a) e anomalia (b) de sistemas frontais identificados para o mês de dezembro de 2014 nos dados da Reanálise I do NCEP/NCAR

A figura 3 mostra o rastreamento dos sistemas frontais para os pontos de grade selecionados no litoral (a), interior (b) e centro (c) do continente no mês de dezembro de 2014.

No litoral (figura 3-a) foram identificados 4 sistemas frontais, o mesmo número identificado pelo CPTEC/INPE em sua Síntese Sinótica Mensal (Síntese Sinótica do Mês de dezembro de 2014). O primeiro sistema foi identificado entre os dias 4 e 6 de dezembro de 2014, tendo atingido todos os pontos rastreados, com exceção do ponto mais ao sul no Rio Grande do Sul. O segundo sistema frontal atuou no Brasil apenas no dia 11 e foi identificado apenas sobre o Rio Grande do Sul. O terceiro sistema frontal atuou entre os dias 14 e 16 nos estados de Santa Catarina e Espírito Santo. O último sistema frontal identificado no litoral neste mês foi localizado sobre o continente entre o Rio Grande do Sul e o estado de São Paulo nos dias 22 e 23 de dezembro de 2014. Na Síntese Sinótica do Mês de dezembro de 2014 do CPTEC/INPE, este último sistema também foi identificado, sendo constatado que ele favoreceu a organização de um episódio de ZCOU (Zona de Convergência de Umidade) e provocou acumulados elevados de precipitação no litoral do estado de São Paulo (Síntese Sinótica do Mês de dezembro de 2014). Estes 4 sistemas frontais também foram identificados no interior (figura 3-b) e centro do 
continente (figura 3-c), sendo que o primeiro e o quarto sistema frontal do mês foram os que avançaram para latitudes mais ao norte e também para longitudes mais à oeste.

(a)

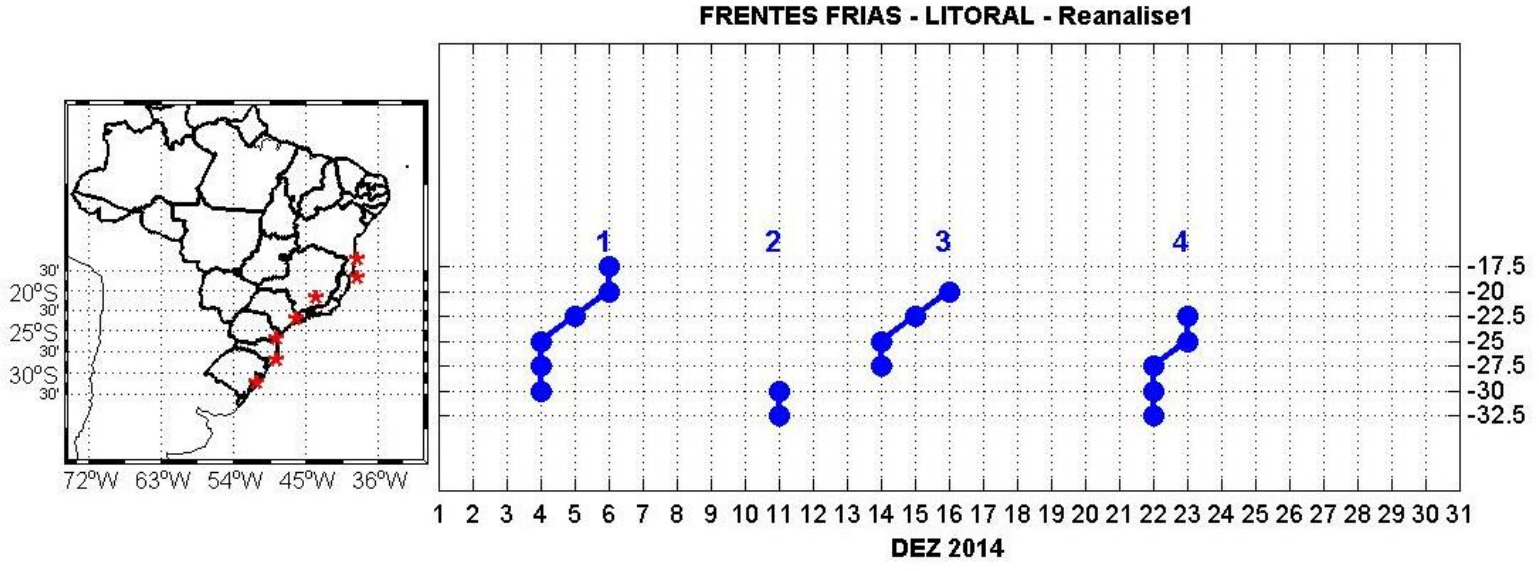

(b)

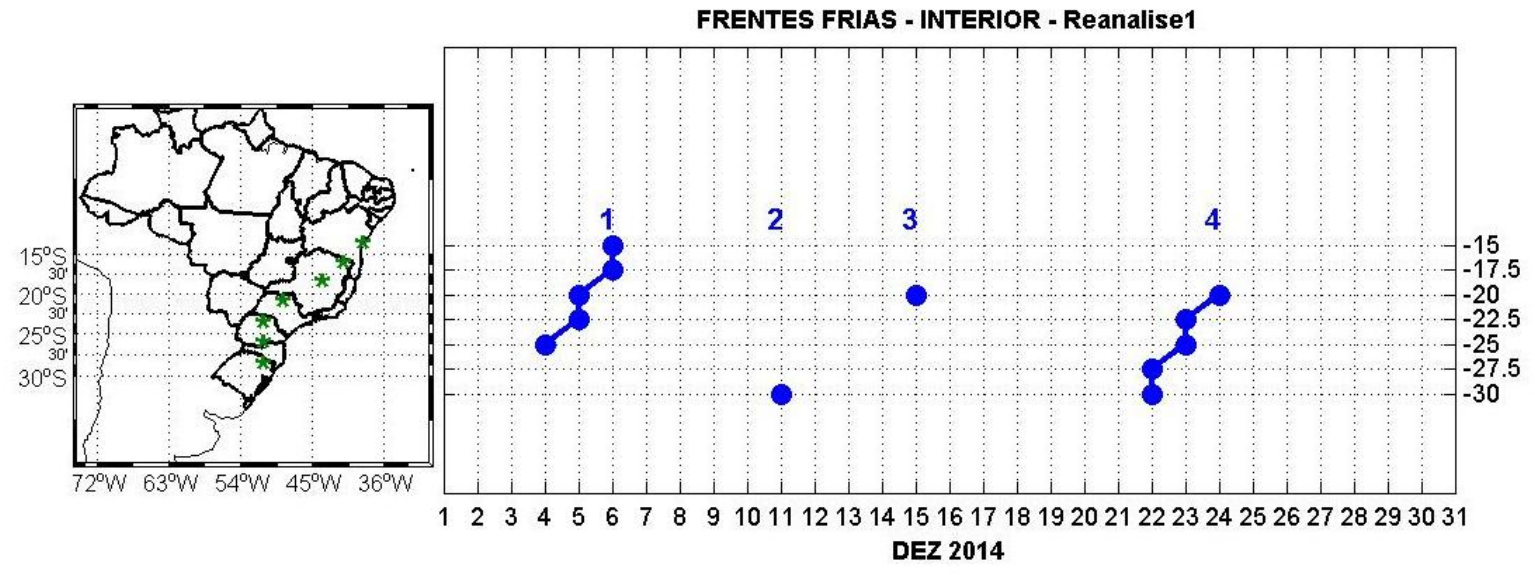

(c)

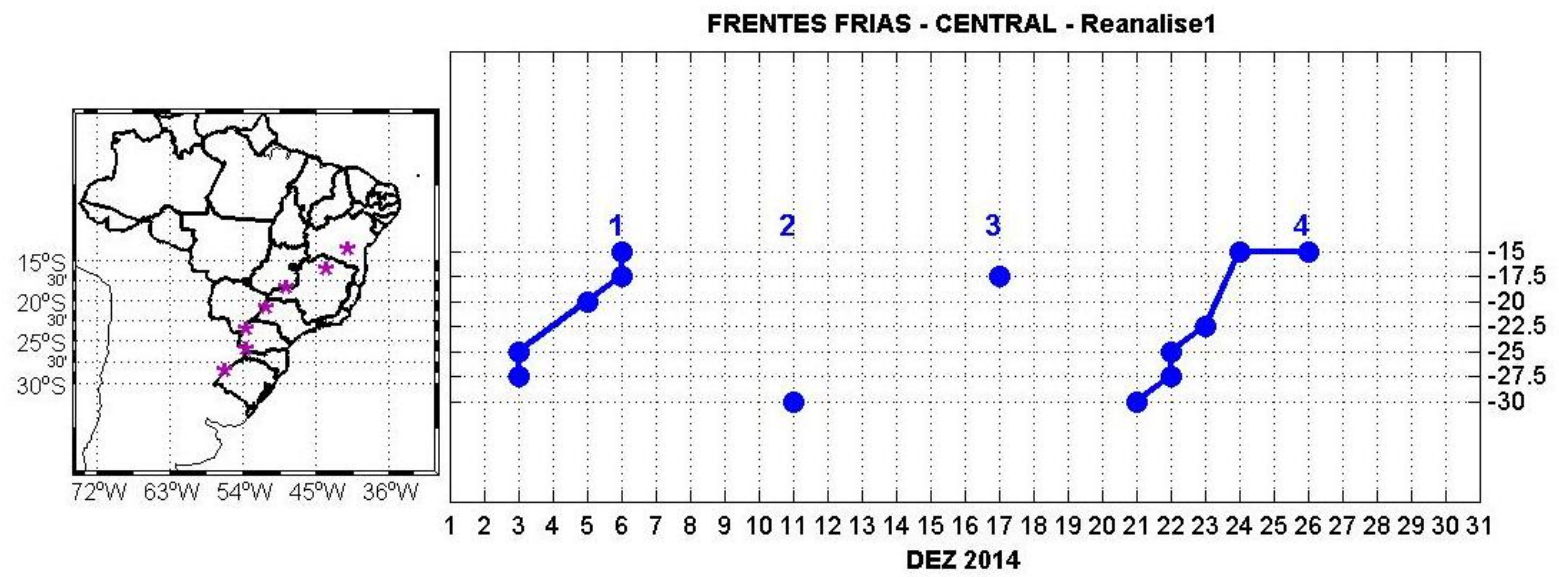

Figura 3 - Sistemas frontais localizados sobre o (a) litoral, (b) interior e (c) centro da América Sul para o mês de dezembro de 2015 nos dados do NCEP/NCAR 


\section{Conclusões}

Este trabalho teve o objetivo de apresentar como é feito o monitoramento mensal dos sistemas frontais para a América do Sul pelo GrEC/USP, usando o mês de dezembro de 2014 como exemplo.

A identificação dos sistemas frontais é baseada em mudanças observadas de um dia para o outro em três condições atmosféricas: queda na temperatura em $925 \mathrm{hPa}$; vento meridional em $925 \mathrm{hPa}$ com mudança na direção (norte para sul); e aumento na PNMM.

Estão disponibilizados no site do grupo (http://www.grec.iag.usp.br) os resultados obtidos através do tipo de análise aqui apresentada, desde janeiro de 2014, na forma de 5 figuras:

- número dos sistemas frontais atuantes na área entre $12.5^{\circ} \mathrm{S}-60^{\circ} \mathrm{S}$ e $90^{\circ} \mathrm{W}-2.5^{\circ} \mathrm{W}$;

- anomalia no número de sistemas frontais para a área entre $12.5^{\circ} \mathrm{S}-60^{\circ} \mathrm{S}$ e $90^{\circ} \mathrm{W}-2.5^{\circ} \mathrm{W}$ baseada na climatologia de 1982-2010 realizada por Pampuch e Ambrizzi (2015);

- data da ocorrência dos sistemas frontais atuantes no mês analisado em 7 pontos do litoral do continente (trajetória dos sistemas sobre o litoral do continente);

- data da ocorrência dos sistemas frontais atuantes no mês analisado em 7 pontos do interior do continente (trajetória dos sistemas sobre o interior do continente);

- data da ocorrência dos sistemas frontais atuantes no mês analisado em 7 pontos do centro do continente (trajetória dos sistemas sobre o centro do continente).

Estes mapas são atualizados mensalmente e formam uma boa base de dados para compreender como é a ocorrência destes sistemas na América do Sul e Oceanos Atlântico e Pacífico adjacentes.

\section{Agradecimentos}

Os autores agradecem ao apoio financeiro da FAPESP (Fundação de Amparo à Pesquisa do Estado de São Paulo) processos no 2011/08191-4, 2012/14316-7 e 2008/58101-9. TA agradece também ao $\mathrm{CNPq}$ pelo financiamento parcial desta pesquisa. LAP agradece ao $\mathrm{CNPq}$ processo 150243/2015-4. Os autores agradecem à Msc.
Lívia Márcia Mosso Dutra, especialista em Laboratório do Departamento de Ciências Atmosféricas, que disponibiliza mensalmente as figuras no site do GrEC/USP.

\section{Referências}

ANDRADE KM. Climatologia e comportamento dos sistemas frontais sobre a América do Sul. 185págs. Dissertação de Mestrado (Mestrado em Meteorologia). 2005. INPE, São José dos Campos.

ANDRADE KM, CAVALCANTI IFA. Climatologia dos sistemas frontais e padrões de comportamento para o verão na América do Sul. In: Congresso Brasileiro de Meteorologia, 13. 2004. Fortaleza- CE. Anais SBMET.

BERRY G, REEDER MJ, JAKOB C. A global climatology of atmospheric fronts. Geophys. Res. Lett. 2011. 38, L04809, doi:10.1029/2010GL046451.

CAVALCANTI IFA, KOUSKY VE. Climatology of South American cold fronts. In: International Conference on Southern Hemisphere Meteorology and Oceanography, 7., Wellington, New Zealand, 2003. Proceedings... New Zealand: American Meteorological Society.

CAVALCANTI IFA, KOUSKY VE. Frentes Frias sobre o Brasil. In: Cavalcanti, I. F. A., Ferreira, N. J., Justi da Silva, M. G. A.; Silva dias, M. A. F. Tempo e clima no Brasil. 2009. São Paulo: Oficina de textos. Cap. 9, p. 133-147.

FEDOROVA N. Meteorologia sinótica. 1999. Pelotas: UFPEL, v.1.

Kalnay E, Kanamitsu M, Kirtler R, Collins W, Deaven D, Gandin L, Iredell M, Saha S, White G, Woollen J, Zhu Y, Chelliah M, Ebisuzaki W, Higgins W, Janowiak J, Mo KC, Ropelewski C, Wang J, Leetma A, Reynolds R, Jenne R, Joseph D. The NCEP/NCAR 40-year reanalysis project. Bull. Amer. Meteorol. Soc. 1996. 77: 437-471.

Notas do Boletim Climanálise http://climanalise.cptec.inpe.br/ rclimanl/boleti m/notas_0308.shtml (Acessado em 01/08/2015)

PAMPUCH LA, AMBRIZZI T. Sistemas Frontais sobre a América do Sul Parte I: Climatologia e intervalo de passagem em dados da Reanálise I do NCEP/NCAR. 2015. Anais do IX Workshop Brasileiro de Micrometeorologia, Santa Maria RS. Em publicação. 